\title{
Regional Anesthesia: Boon for Chronic Kidney Disease Patients Undergoing Vascular Access Surgery
}

\author{
Prapti Rath ${ }^{1}$ Archana Shivashankar ${ }^{1} \quad$ Luv Luthra $^{2} \quad$ Nivedita Mitta $^{2}$ \\ ${ }^{1}$ Department of Anaesthesia, MS Ramaiah Medical College, \\ Bengaluru, India \\ ${ }^{2}$ Department of Vascular Surgery, MS Ramaiah Medical College, \\ Bengaluru, India

\begin{abstract}
Address for correspondence Prapti Rath, MD, Department of Anaesthesia, MS Ramaiah Medical College, F-32, Sriram Sadhana, Gokula, Mathikere, Bengaluru 560054, India (e-mail: drpraptirath@gmail.com).
\end{abstract}

Int J Recent Surg Med Sci 2019;5:37-41
Abstract
Keywords
- chronic kidney disease
- vascular access
- general anesthesia
- regional anesthesia

The incidence of chronic kidney disease (CKD) is alarmingly high in Indian population with a steep rise in end-stage renal disease patients requiring dialysis access. The preexisting comorbidities associated with high morbidity further necessitate an anesthetic plan which provides benefits intraoperatively as well postoperatively. Different anesthesia techniques can be employed in CKD patients which are associated with complications. The aim of this review is to study the role and benefits of regional anesthesia in CKD patients.

\section{Introduction}

Diabetes and hypertension are the most common primary causes of end-stage renal disease (ESRD). Patients who undergo procedures for arteriovenous access have multiple comorbidities. These comorbidities may have specific anesthetic implications. This article focuses on the anesthetic considerations throughout the perioperative period with special emphasis on the role of regional anesthesia.

During the preoperative period, the patients must be optimized with a system-based approach, ensuring all the comorbidities are addressed and treated prior to surgery. The type of anesthesia is determined with the common decision of an anesthesiologist, surgeon, and keeping in mind the general condition of the patient. ${ }^{1}$

\section{Decision to Offer Dialysis}

Classification of chronic kidney disease (CKD) has evolved and it is defined according to the updated 2015 National Institute for Health and Care Excellence (NICE) guidelines. ${ }^{2}$ It was previously defined as glomerular filtration rate (GFR) $<60 \mathrm{~mL} / \mathrm{min} / 1.73 \mathrm{~m}^{2}$ for 3 months or more, or abnormalities of renal function or structure, ${ }^{3}$ but as per the NICE guidelines, the grades of CKD are based on GFR and albumin:creatinine ratio. Patients with grade 5 CKD, or less severe are the candidates for dialysis.

\section{Types of Anesthesia}

\section{Local Anesthesia}

Local anesthesia (LA) is a simple technique, but is associated with higher risks of local edema ${ }^{4,5}$ and vessel spasms. ${ }^{5,6}$ In case the procedures last long, repeated LA injections or additional sedative medications may be required. These can lead to side effects based on the drugs used.

\section{Regional Anesthesia}

Upper extremity is the most common site for placement of vascular access, rarely lower extremities are used. Regional anesthesia (RA) of the upper extremity requires blockade of the brachial plexus at different levels (supraclavicular, infraclavicular, axillary) depending on the site of surgery. The advantage of regional anesthesia over other techniques is that it avoids airway manipulation, decreased drug side effects as seen in general anesthesia (GA) and minimum alveolar concentration. Patients are hemodynamically stable during surgery, has better postoperative analgesia, and faster recovery. ${ }^{7-10}$ There is a documented evidence that RA reduces vasospasm and causes vasodilation to maintain a higher blood flow during the perioperative period. ${ }^{11,12}$ The risks associated with RA include neuropathy from mechanical trauma to the nerves, hematoma, infection, neurotoxicity due to direct intraneuronal injection of the agent,
License terms

10.1055/s-0040-1701373

ISSN 2455-7420. 
neuronal ischemia, and local anesthetic toxicity by the accidental injection of anesthetic agent into the vessel.

The main challenge is to administer RA in patients with variations in anatomical landmarks. ${ }^{13}$ Traditional techniques for nerve localization (i.e., paresthesia and nerve stimulation) depend upon anatomy based on the surface landmarks. For obese patients and those with variations in anatomy, the administration of RA is a challenging job due to difficulty in identification of surface landmarks and predicting the depth of the nerve to be blocked. Motor response during and after nerve stimulation does not exclude the possibility of block failure. ${ }^{10}$ Ultrasound-guided technique to administer block is the most preferred technique as it helps in direct visualization of the nerves and vessels. It provides clear images of the needle location and spread of injected local anesthetic agent. ${ }^{10,14}$ It reduces the incidence of systemic local anesthetic toxicity as the drug is injected in close proximity to the nerve, thus minimizing the dose of the drug. ${ }^{15}$ Several randomized trials reported fewer needle passes, faster block performance, and faster onset of the block with ultrasonography (USG) use compared with the conventional blind technique. $^{16}$

\section{General Anesthesia}

The indications of GA are the patients having difficult anatomy, in whom RA is contraindicated or in patients in whom RA was attempted but failed. It enhances the blood flow due to vasodilatory effect of anesthetics, but this effect is limited to the intraoperative period. ${ }^{4} \mathrm{GA}$ can lead to increased stress response, hemodynamic instability, potential drug interaction, ${ }^{5,6,17}$ residual muscle relaxant, respiratory depression during postoperative period, and poor postoperative pain control. However, with careful management, the risk of these possible complications is low. ${ }^{18}$

Types of regional anesthesia for ESRD patients

For successful ultrasound (US)-guided nerve block, the performer should

1. Identify the target nerve structure and obtain appropriate image.

2. Track the in-plane needle advancement in real time.

3. Assess the LA spread around the target nerve (-Fig. 1).

Ultrasound-guided supraclavicular block: Patient is positioned supine with head turned to contralateral side and arm is placed on the side of patient. USG machine with linear transducer $(8-18 \mathrm{MHz})$ is used.

The insulated stimulating needle of $50 \mathrm{~mm}$ and 22 gauge is preferred and 20 to $30 \mathrm{~mL}$ of 0.5 to $0.75 \%$ ropivacaine or $0.5 \%$ bupivacaine is used as local anesthetic agent with adjuvants.

\section{Technique}

1. After all sterile and aseptic precautions, and placement of monitors, the skin is prepared with antiseptic solution.

2. The transducer is placed parallel and abutting to the clavicle in the supraclavicular region. The subclavian artery, first rib, pleura, and the hypoechoic nerves of the brachial plexus are identified with USG (-Fig. 2). The transducer is held with a slight vertical tilt aiming into the chest to obtain the transverse images of the subclavian artery and optimal images of plexus. Subclavian artery is identified by moving the probe laterally and medially, as well as toggle the probe from caudad to cephalad. Color Doppler can be used when in doubt. Brachial plexus is usually located lateral and superficial to the artery, with a characteristic

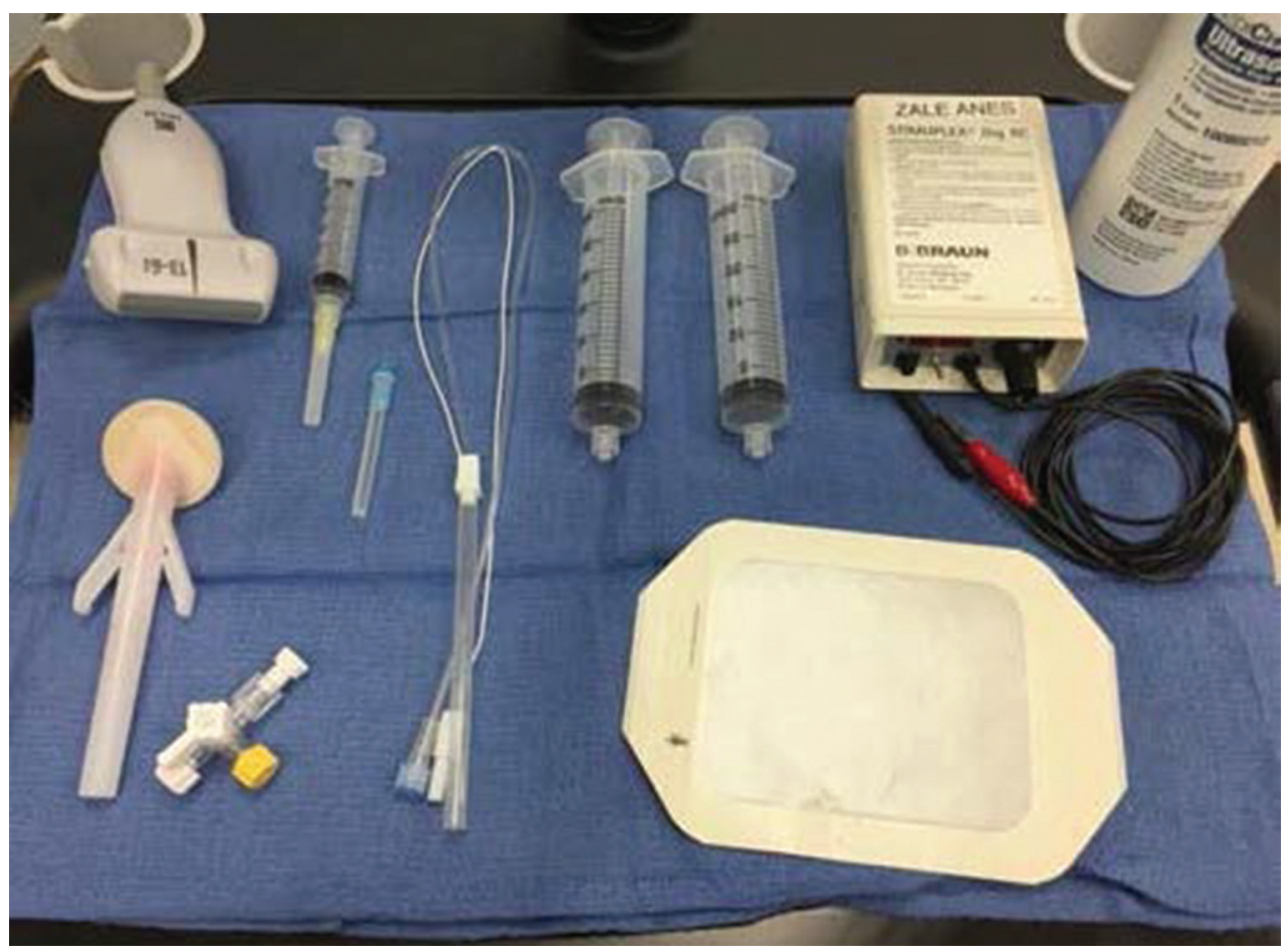

Fig. 1 Basic equipment for regional blocks. 


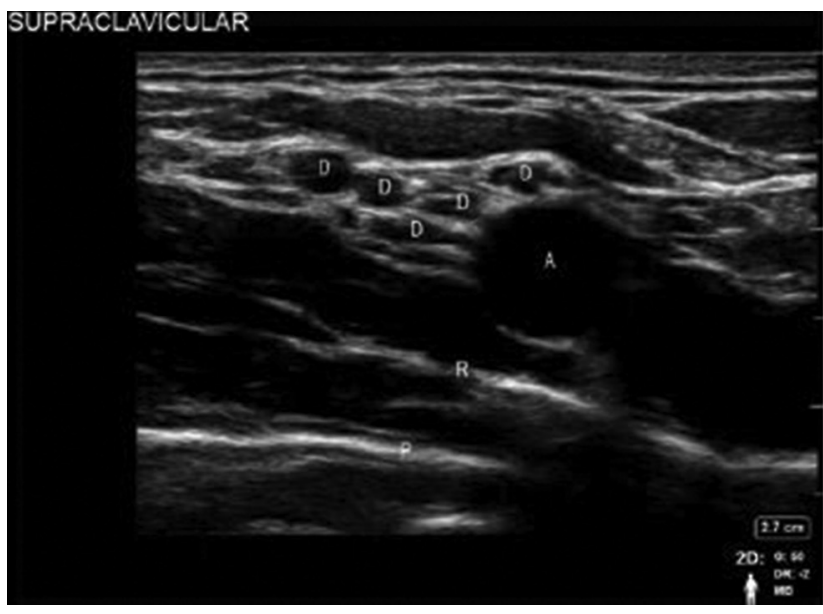

Fig. 2 Sonoanatomy of supraclavicular block with divisions of brachial plexus. A, subclavian artery; D, divisions; P, pleura; R, rib.

"honeycomb" appearance. There are two distinct appearances of the brachial plexus, one as a grape-like cluster of 5 to 6 hypoechoic circles, which represents the divisions of the brachial plexus ( - Fig. 2) or as three hypoechoic structures, which represent the trunks of the brachial plexus ( - Fig. 3). Pleura and first rib are visualized by increasing the depth of US. With the first rib in view directly beneath the brachial plexus, it can be used as a backstop to prevent inadvertent pleural injury. The artery should be visualized at all times while performing the block.

3. The skin on the lateral side of the probe is anesthetized with local anesthetic using a 25 -gauge needle creating a skin wheal after identifying all the anatomical structures under USG guidance.

4. The needle is applied using the in-plane approach from the lateral aspect of the transducer and advanced at a very shallow angle to allow for visualization on US. To avoid pneumothorax the needle tip should always be visible. A pop may be felt when entering the nerve sheath. It is best to first inject at the site immediately adjacent to the artery and inferior to the lower trunk (corner pocket). Prior to every $3-\mathrm{mL}$ injection, aspiration should be done and the

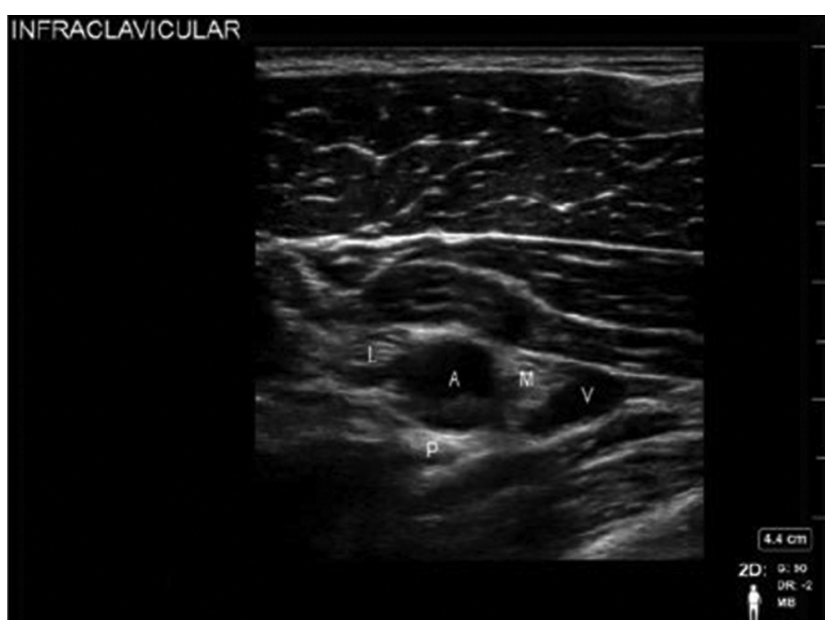

Fig. 3 Sonoanatomy of infraclavicular block. A, axillary artery; I, wwmedial cord; L, lateral cord; P, posterior cord; V, axillary vein. spread of LA observed. Injection should be stopped in case patient complains of pain, paresthesia going down to the extremity, or resistance is met upon injection. In this situation, withdraw slightly, aspirate, and reattempt injection.

After entering the correct position, 5 to $10 \mathrm{~mL}$ of anesthetic agent is injected in one given location.

\section{Ultrasound-Guided Infraclavicular Block}

Patient position: Supine with head slightly turned contralateral side. Arm is abducted 90 degrees, externally rotated, and flexed at the elbow. This maneuver rotates the clavicle posteriorly while also moving the plexus into a more superficial position.

Transducer: High frequency transducer may not be adequate due to deeper location of the brachial plexus at this level in overweight patients. Lower frequency transducer $(5-12 \mathrm{MHz})$ may be required in the infraclavicular region to image cords of the brachial plexus that are deep $(5 \mathrm{~cm}$ or more). A smaller footprint ( $25 \mathrm{~mm}$ ) may also be considered because the infraclavicular fossa is small and the larger footprint probe can hang over the clavicle.

Needle size: $100 \mathrm{~mm}, 22$-gauge blunt insulated stimulating needle.

Local anesthetic: 20 to $30 \mathrm{~mL}$ of 0.5 to $0.75 \%$ ropivacaine or $0.5 \%$ bupivacaine with adjuvants.

\section{Technique}

1. Patient is prepared under all aseptic conditions.

2. Ultrasound probe is directly placed inferior to the clavicle in parasagittal orientation in the infraclavicular fossa. The infraclavicular fossa is a natural depression approximately $1 \mathrm{~cm}$ medial to the coracoid process of the scapula.

The pectoralis major muscle, pectoralis minor muscle, axillary artery and vein, and the hyperechoic cords of the brachial plexus which lie lateral, medial, and posterior to the artery at 3, 6, and 9 o'clock positions are the key structures to be identified ( $\boldsymbol{- \text { Fig. }} \mathbf{3}$ ). The transducer may be moved laterally, medially, or toggled to provide the optimal images of the nerves and vessels. To minimize the risk of pleural breach, once an image is obtained, rotate the transducer's caudal edge slightly away from the midline. This small detail will still provide an excellent view of the brachial plexus while steering the needle's trajectory into the periphery of the axilla rather than the thorax.

3. After establishing the ideal image, the skin on the superior side of the probe is anesthetized with local anesthetic using a 25-gauge needle creating a skin wheal.

4. The needle is inserted from either the inferior or superior side of the transducer using in-plane approach. As the needle is introduced, the transducer is adjusted to obtain a view of the tip during its progress. It is essential to maintain imaging of the tip of the needle at all times to avoid vascular or pleural puncture. The needle is then advanced under continuous observation toward each of the cords.

Local anesthetic of 8 to $10 \mathrm{~mL}$ is then injected next to each cord. It is easy to guide the needle to the lateral cord first, using the nerve stimulation if desired for confirmation 


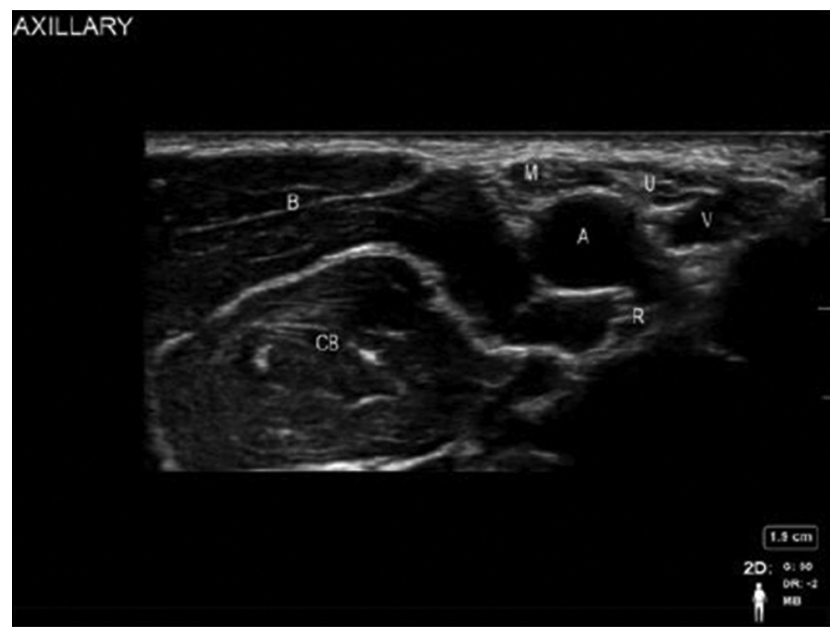

Fig. 4 Sonoanatomy of axillary block. A, axillary artery; B, biceps; CB, coracobrachialis; $M$, median nerve; $R$, radial nerve; $U$, ulnar nerve; $V$, axillary vein.

with stimulation of the median or musculocutaneous nerves. Direct needling of the medial cord is usually not necessary nor recommended due to its precarious position. With each needle positioning, the anesthetist may utilize peripheral nerve stimulation to confirm the target. Nerve stimulation is helpful in this block because the cords are deeper than the more proximal brachial plexus and harder to visualize.

\section{Ultrasound-Guided Axillary Block}

Patient is positioned supine with head slightly turned to contralateral side. Arm is abducted, externally rotated, and flexed at the elbow.

Transducer: High frequency 38-mm transducer.

Needle size: $50 \mathrm{~mm}, 22$-gauge blunt stimulating needle.

Local anesthetic: 25 to $30 \mathrm{~mL}$ of 0.5 to $0.75 \%$ ropivacaine or $0.5 \%$ bupivacaine with adjuvants.

\section{Technique}

1. Monitors are connected and patient is sedated with supplemental oxygen. Skin is prepared with antiseptic solution.

2. Ultrasound probe is placed in the axilla at the crease formed by the pectoralis major and biceps muscles, perpendicular to the axillary artery.

Axillary artery and vein and the hyperechoic terminal nerves of the brachial plexus: median, ulnar, and radial which lie superior, inferior, and posterior to the artery are the anatomical structures to be identified. Color Doppler is used to identify the vascular structure in this region. The median nerve tends to appear consistently at 12 o'clock position with the ulnar nerve between 2 and 5 o'clock positions and radial nerve between 4 and 9 o'clock positions ( - Fig. 4). To image the musculocutaneous nerve, move the transducer slightly superior toward the biceps muscle from the axilla. The nerve may be viewed either in the plane between the coracobrachialis muscle and the biceps muscle or in the body of the coracobrachialis muscle. The nerve is hyperechoic, brighter than the surrounding muscle.

3. After establishing the ideal image, the skin is anesthetized with local anesthetic using a 25-gauge needle creating a skin wheal.

4. Initially, the needle is inserted from the superior side of the transducer using in-plane approach toward the median nerve. Local anesthetic is incrementally injected $(6-8 \mathrm{~mL})$ until a halo appears around the nerve. Then direct the needle to the ulnar nerve to the inferior edge of the artery and LA is injected as described above. Next, the needle is redirected more posterior, and guided to the radial nerve, followed by incremental injection of 6 to $8 \mathrm{~mL}$ of LA ( - Fig. 4). To block the musculocutaneous nerve, the transducer is moved toward the biceps muscle, and the needle is placed in-plane superior to the probe; 6 to $8 \mathrm{~mL}$ of $\mathrm{LA}$ is injected until a halo is seen around the nerve. In addition to USG, nerve stimulation can also be used to identify nerve and artery in patients with variable anatomical position.

\section{Conclusion}

RA is very useful and safe in patients with CKD as it not only reduces the exposure of patient to many anesthetic drugs, it also decreases the risks associated with GA. There is significant evidence that vasodilation, greater fistula blood flow, sympathectomy like effects not only assist the surgeon intraoperatively but also maintain graft patency hours after the surgery.

CKD patients have associated comorbidities and RA has proven to be a boon for such patients due to its better and safe techniques of administration, minimal use of drugs, shorter duration, good postoperative analgesia, and faster recovery.

\section{Conflict of Interest}

None declared.

\section{References}

1 Nguyen H, Pai SL, Thammasithiboon S, et al. Anesthetic considerations for vascular access placement in patients with end stage renal disease. SM J Anesth 2017;3(1):1010

2 National Institute for Health and Clinical Excellence. Chronic kidney disease in adults: assessment and management. 2014. Available at: nice.org.uk/guidance/cg182. Accessed September 14,2016

3 Levey AS, Eckardt KU, Tsukamoto Y, et al. Definition and classification of chronic kidney disease: a position statement from kidney disease: improving global outcomes (KDIGO). Kidney Int 2005;67(6):2089-2100

4 Malinzak EB, Gan TJ. Regional anesthesia for vascular access surgery. Anesth Analg 2009;109(3):976-980

5 Konner K, Nonnast-Daniel B, Ritz E. The arteriovenous fistula. J Am Soc Nephrol 2003;14(6):1669-1680

6 Hingorani AP, Ascher E, Gupta P, et al. Regional anesthesia: preferred technique for venodilatation in the creation of upper extremity arteriovenous fistulae. Vascular 2006;14(1):23-26

7 Rodríguez J, Quintela O, López-Rivadulla M, Bárcena M, Diz C, Alvarez J. High doses of mepivacaine for brachial plexus block in patients with end-stage chronic renal failure. A pilot study. Eur J Anaesthesiol 2001;18(3):171-176 
8 Weissman C. The metabolic response to stress: an overview and update. Anesthesiology 1990;73(2):308-327

9 Seltzer JL. Is regional anesthesia preferable to general anesthesia for outpatient surgical procedures on an upper extremity? Mayo Clin Proc 1991;66(5):544-547

10 Sites BD, Brull R. Ultrasound guidance in peripheral regional anesthesia: philosophy, evidence-based medicine, and techniques. Curr Opin Anaesthesiol 2006;19(6):630-639

11 Shemesh D, Olsha O, Orkin D, et al. Sympathectomy-like effects of brachial plexus block in arteriovenous access surgery. Ultrasound Med Biol 2006;32(6):817-822

12 Laskowski IA, Muhs B, Rockman CR, et al. Regional nerve block allows for optimization of planning in the creation of arteriovenous access for hemodialysis by improving superficial venous dilatation. Ann Vasc Surg 2007;21(6):730-733

13 Peng PW, Narouze S. Ultrasound-guided interventional procedures in pain medicine: a review of anatomy, sonoanatomy, and procedures: part I: nonaxial structures. Reg Anesth Pain Med 2009;34(5):458-474

14 Gray AT. Ultrasound-guided regional anesthesia: current state of the art. Anesthesiology 2006;104(2):368-373, discussion 5A

15 Marhofer P, Chan VW. Ultrasound-guided regional anesthesia: current concepts and future trends. Anesth Analg 2007;104(5):1265-1269

16 Liu SS, Ngeow JE, Yadeau JT. Ultrasound-guided regional anesthesia and analgesia: a qualitative systematic review. Reg Anesth Pain Med 2009;34(1):47-59

17 Viscomi CM, Reese J, Rathmell JP. Medial and lateral antebrachial cutaneous nerve blocks: an easily learned regional anesthetic for forearm arteriovenous fistula surgery. Reg Anesth 1996;21(1):2-5

18 Rang ST, West NL, Howard J, Cousins J. Anaesthesia for chronic renal disease and renal transplantation. EAU-EBU Update Ser 2006;4(6):246-256 